\title{
Time dependence of multi-ion absorption into human enamel from surface pre- reacted glass-ionomer (S-PRG) filler eluate
}

\author{
Ayumi OGAWA ${ }^{1 *}$, Takahiro WADA ${ }^{2 *}$, Yoshiyuki MORI ${ }^{1}$ and Motohiro UO 2,3 \\ ${ }^{1}$ Department of Dentistry, Oral and Maxillofacial Surgery, Jichi Medical University, 3311-1 Yakushiji, Shimotsuke, Tochigi 329-0498, Japan \\ ${ }^{2}$ Department of Advanced Biomaterials, Graduate School of Medical and Dental Sciences, Tokyo Medical and Dental University, 1-5-45, Yushima, \\ Bunkyo-ku, Tokyo 113-8549, Japan \\ ${ }^{3}$ Department of Materials Engineering, Graduate School of Engineering, The University of Tokyo, 7-3-1, Hongo, Bunkyo-ku, Tokyo 113-8656, Japan \\ Corresponding author, Motohiro UO; E-mail: uo.abm@tmd.ac.jp
}

\begin{abstract}
Ion incorporation into the tooth is expected to be effective for caries prevention. Time-dependent ion incorporation released from surface pre-reacted glass-ionomer (S-PRG) filler eluate into tooth enamel was estimated by using inductively coupled plasma atomic emission spectroscopy (ICP-AES). Al, B, and Sr contents in enamel were increased in a time-dependent manner with immersion in S-PRG eluate. Clear ion incorporation was observed after $1 \mathrm{~h}$ of immersion in S-PRG filler eluate. Sr showed remarkable incorporation in enamel —up to 7,900 ppm - after 28 days of immersion. Sr and B incorporation rapidly occurred in S-PRG filler eluate, compared with their single component solutions. Simultaneous incorporation of cations and anions from S-PRG eluate occurred under balanced charge and may assist in rapid ion incorporation. Thus, various useful ions could be effectively incorporated into tooth enamel by applying S-PRG filler or its eluate; a bioactive effect can be expected.
\end{abstract}

Keywords: Ion absorption, Time dependence, Surface pre-reacted glass-ionomer filler, Strontium, Elemental analysis

\section{INTRODUCTION}

Mineral loss in dental caries is closely related to the mineral content in the tooth. Fluoride (F) incorporation into tooth is a well-known effective method for caries prevention ${ }^{1,2}$. Strontium $(\mathrm{Sr})$ is a cation species that can be incorporated in hydroxyapatite (HAP) in natural teeth. A relationship has been reported between high Sr content in enamel and a low incidence of caries ${ }^{3,4}$. Therefore, ion incorporation into the tooth, including fluoride, as well as Sr and other ions, may be effective for caries prevention.

Surface pre-reacted glass-ionomer (S-PRG) fillers release multiple ions (e.g., fluoride, borate, silicate, aluminum, sodium, and strontium) ${ }^{5}$; various bioactive effects by these released ions have been reported for the suppression of bacterial adhesion ${ }^{6-9}$, biofilm formation ${ }^{10,11)}$, prevention of demineralization ${ }^{12-14)}$, and enhancement of remineralization ${ }^{15,16)}$. As noted above, $\mathrm{Sr}$ and $\mathrm{F}$ ions are expected to provide a preventive effect with respect to caries. In addition, borate $\left(\mathrm{BO}_{3}{ }^{3-}\right)$ and $\mathrm{F}$ ions are suspected to exhibit antibacterial effects ${ }^{8,9}$. Therefore, the depth of incorporation and chemical state of the ions released from S-PRG fillers into teeth have been studied by various methods to investigate their bioactive effects.

Han and Okiji reported the incorporation of $\mathrm{Sr}$ and $\mathrm{F}$ into dentin in contact with an S-PRG fillercontaining sealer for up to 90 days by using electron probe micro analysis (EPMA) ${ }^{17}$. Funato also estimated the incorporation of $\mathrm{Sr}$ and $\mathrm{F}$ into enamel in contact with S-PRG-containing cavity varnish for 5 weeks, in

*Authors who contributed equally to this work. Received Sep 21, 2018: Accepted Nov 12, 2018 doi:10.4012/dmj.2018-314 JOI JST.JSTAGE/dmj/2018-314 a study using particle-induced X-ray ( $\gamma$ ray) emission (PIXE/PIGE) ${ }^{18}$. In both cases, $\mathrm{Sr}$ and F penetration into dentin and enamel from the interface with the S-PRGcontaining material occurred for an extended period. Notably, Sr was reported to accumulate in the enamel and dentin for 3 months with S-PRG eluate immersion; this accumulated $\mathrm{Sr}$ was speculated to incorporated to the Ca site of HAP ${ }^{19}$. By using PIXE, Nakamura et al. revealed $\mathrm{Sr}$ enrichment on the enamel surface after 40 min of contact with an S-PRG-containing experimental toothpaste ${ }^{14)}$; their report suggested that early $\mathrm{Sr}$ incorporation could occur in less than $1 \mathrm{~h}$, and that the acid resistance of enamel was improved. Therefore, the effect of ion incorporation released from S-PRG filler could be expected within a short period. However, the incorporation of other ions has not been established. In order to estimate the effect of multiple ions released from S-PRG filler, the time-dependent behavior of the incorporation of these multiple ions should be evaluated.

In this study, the incorporation of multiple ions from the S-PRG filler eluate (S-PRG eluate) into human enamel powder during various immersion periods $(1 \mathrm{~h}$ to 4 weeks) was evaluated by using inductively coupled plasma atomic emission spectroscopy (ICP-AES). In addition, the effect of simultaneous incorporation of multiple ions was estimated by comparison with a single-ion solution of Sr and borate.

\section{MATERIALS AND METHODS}

\section{Human enamel powder preparation}

Approximately 100 extracted human molars were collected. Caries were checked by visual inspection, and 
then removed carefully. The molar was sliced into 1-mmthick sections and enamel was carefully separated. Crude granules of enamel were finely ground by mortar into a homogeneous powder and sieved to $<75 \mu \mathrm{m}$. The study protocol was approved by the Ethical Committee of Jichi Medical University Hospital.

\section{Preparation of S-PRG eluate and other solutions}

To simulate ion release from S-PRG fillers into the water medium, S-PRG filler eluate was prepared by mixing equal portions of S-PRG filler as in a previous report ${ }^{5)}$. Strontium and borate solutions ("Sr solution" and "B solution," respectively) were prepared with reagent-grade $\mathrm{SrCl}_{2} \cdot 6 \mathrm{H}_{2} \mathrm{O}$ and $\mathrm{B}_{2} \mathrm{O}_{3}$ (Kanto Chemical, Tokyo, Japan) dissolved in ultra-pure water (Simplicity UV, Merck, Darmstadt, Germany). Ion contents in these solutions were quantified by using ICP-AES (Spectro Arcos, Hitachi High-technologies, Tokyo, Japan). The standard solutions for ICP-AES analyses comprised multi-element standard solution (100 ppm, XSTC-22, Seishin Trading, Kobe, Japan) and Sr standard solution (1,000 ppm, Nakarai Tesque, Kyoto, Japan). Sr and borate contents in the $\mathrm{Sr}$ and $\mathrm{B}$ solutions were adjusted to a level similar to those in the S-PRG eluate, as shown in Table 1.

\section{Immersion of enamel powder in S-PRG filler eluate and} other solutions

A total of $0.2 \mathrm{~g}$ of enamel powder was immersed in $8 \mathrm{~mL}$ each of S-PRG eluate, Sr solution, and B solution, using polymer tubes. The tubes were sealed and maintained at $37^{\circ} \mathrm{C}$ with gentle stirring by using a rotary mixer (MR-3, As-One, Tokyo, Japan) for 1, 2, and 8 h, as well as 1, 3, 7, 14 , and 28 days. The immersing solutions were replaced with fresh solutions after 1,3 , and 7 days of incubation, to avoid exhaustion of the solute ions by absorption. After immersion, the enamel powder was collected by centrifugation and washed 3 times with ultra-pure water, then dried.

Quantitative analyses of element concentrations in enamel powder

A total of $0.1 \mathrm{~g}$ of enamel powder was accurately weighed and dissolved in 3M HCL (aqueous) (Ultra-pur 100, Kanto Chemical, Tokyo, Japan) in a polymer test tube at $60^{\circ} \mathrm{C}$ overnight. The dissolved solution was diluted in a volumetric flask and subjected to ICP-AES analysis. The conditions of ICP-AES analysis were as described above.
Surrounding structure analysis of $\mathrm{Sr}$ by the extended $X$-ray absorption fine structure (EXAFS) measurement Approximately $0.1 \mathrm{~g}$ each of S-PRG-immersed enamel (1 day) was subjected to EXAFS measurement. Sr K-edge XAFS spectra were measured on NW-10A beamlines at the Photon Factory of the High Energy Accelerator Research Organization (Tsukuba, Japan) by using the transmission method. Measurement and EXAFS analysis were described in the previous report ${ }^{19)}$.

\section{RESULTS}

Time dependence of the accumulation of various ions in enamel

Table 2 shows the time dependence of $\mathrm{B}, \mathrm{Na}, \mathrm{Mg}, \mathrm{Al}$, $\mathrm{Zn}$, and Sr content in natural enamel and in enamels immersed in S-PRG eluate for various periods, up to 28 days; these time-dependence data are also plotted in Fig. $1 \mathrm{~A}(\mathrm{Al}, \mathrm{B}$, and $\mathrm{Sr})$ and Fig. $1 \mathrm{~B}(\mathrm{Mg}, \mathrm{Na}$, and $\mathrm{Zn})$. $\mathrm{Mg}, \mathrm{Na}, \mathrm{Zn}$, and $\mathrm{Sr}$ are intrinsic elements in natural enamel, while $\mathrm{Al}$ and $\mathrm{B}$ are rarely present. As shown in Fig. 1A, Al, B, and Sr contents in enamel were increased in a logarithmic manner dependent upon immersion in S-PRG eluate. Absorption of those ions occurred quickly; half of the final concentration (i.e., half of the concentration at 28 days) was absorbed with $2 \mathrm{~h}$ of immersion. In contrast, $\mathrm{Mg}, \mathrm{Na}$, and $\mathrm{Zn}$ were not changed by immersion, as shown in Fig. $1 \mathrm{~B}$. $\mathrm{Mg}$ and $\mathrm{Zn}$ were not present in S-PRG eluate; thus, their concentrations in immersed enamel did not change with respect to their natural levels. Na was present in the eluate $(277 \mathrm{ppm})$; however, it was also present in the enamel in high concentrations (7,000 ppm in approx.), such that there was no effect of S-PRG eluate immersion.

Comparison of S-PRG eluate and the $\mathrm{SrCl}_{2}$ and $\mathrm{B}_{2} \mathrm{O}_{3}$ aqueous solutions

Figure 2 and Table 3 show the comparison of $\mathrm{Sr}$ and $\mathrm{B}$ contents on the basis of immersion in S-PRG eluate and the Sr and B solutions. Both Sr and B were present in the enamel at significantly greater concentrations after immersion in S-PRG eluate, compared with immersion in the simple aqueous solutions with content of $\mathrm{Sr}$ and $\mathrm{B}$.

\section{Surrounding structure analysis of Sr by EXAFS}

The radial distribution function (RDF) around $\mathrm{Sr}$ in enamel immersed in S-PRG eluate for 1 day is shown in Fig. 3; RDF represents a function of the distances between the center atom ( $\mathrm{Sr}$ ) and neighboring atoms,

Table 1 Concentration of Al, B, Na, and Sr in the S-PRG eluate, Sr solution, and B solution estimated with ICP-AES

\begin{tabular}{ccccc}
\hline Solution & \multicolumn{4}{c}{ Concentration of element (ppm) } \\
\cline { 2 - 5 } & $\mathrm{Al}$ & $\mathrm{B}$ & $\mathrm{Na}$ & $\mathrm{Sr}$ \\
\hline S-PRG eluate & 25.8 & 1,680 & -149 & 277 \\
Sr solution & - & - & - & - \\
B solution & - & 1,690 & - \\
\hline
\end{tabular}


Table 2 Concentration of elements contained in the enamel before and after immersion into S-PRG eluate for various periods (standard deviation is indicated in parentheses)

\begin{tabular}{lcccccc}
\hline \multirow{2}{*}{ Immersion period } & \multicolumn{7}{c}{ Concentration of element (ppm) } \\
\cline { 2 - 6 } & $\mathrm{Al}$ & $\mathrm{B}$ & $\mathrm{Mg}$ & $\mathrm{Na}$ & $\mathrm{Zn}$ & $\mathrm{Sr}$ \\
\hline Before immersion & $52.4(3.3)$ & $1.6(0.4)$ & $2,630(19)$ & $7,150(66)$ & $263(10)$ & $121(1)$ \\
$1 \mathrm{~h}$ & $237(12)$ & $254(27)$ & $2,330(31)$ & $7,580(243)$ & $294(5)$ & $2,880(138)$ \\
$2 \mathrm{~h}$ & $483(10)$ & $193(14)$ & $2,680(14)$ & $6,930(96)$ & $283(4)$ & $3,550(30)$ \\
$8 \mathrm{~h}$ & $432(25)$ & $290(10)$ & $2,610(17)$ & $6,710(86)$ & $193(2)$ & $3,890(108)$ \\
1 day & $664(40)$ & $301(26)$ & $2,700(31)$ & $8,020(112)$ & $436(7)$ & $4,870(186)$ \\
3 days & $1,050(42)$ & $386(16)$ & $2,860(28)$ & $7,230(126)$ & $208(7)$ & $5,980(188)$ \\
7 days & $1,050(15)$ & $392(11)$ & $2,920(8)$ & $6,390(71)$ & $302(3)$ & $6,350(76)$ \\
28 days & $1,350(66)$ & $550(14)$ & $2,940(20)$ & $7,950(100)$ & $245(3)$ & $7,900(255)$ \\
\hline
\end{tabular}
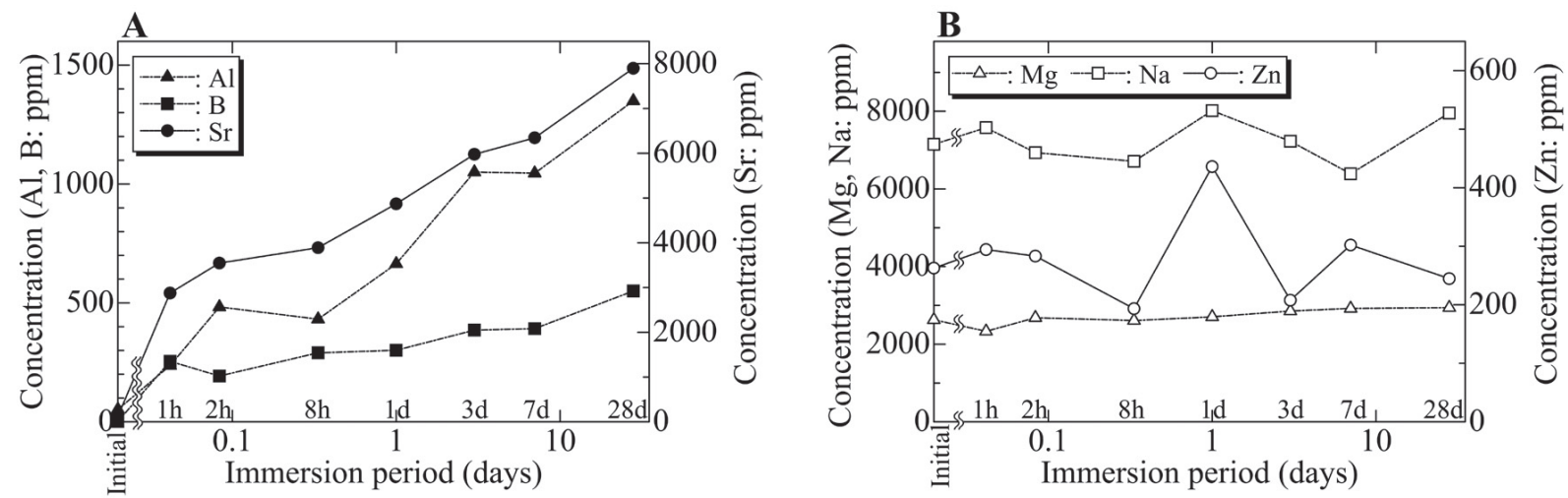

Fig. 1 Dependence of elemental concentrations in enamel on the immersion period (logarithmic) in S-PRG eluate. A: Al, B, and $\mathrm{Sr}, \mathrm{B}: \mathrm{Mg}, \mathrm{Na}$, and $\mathrm{Zn}$

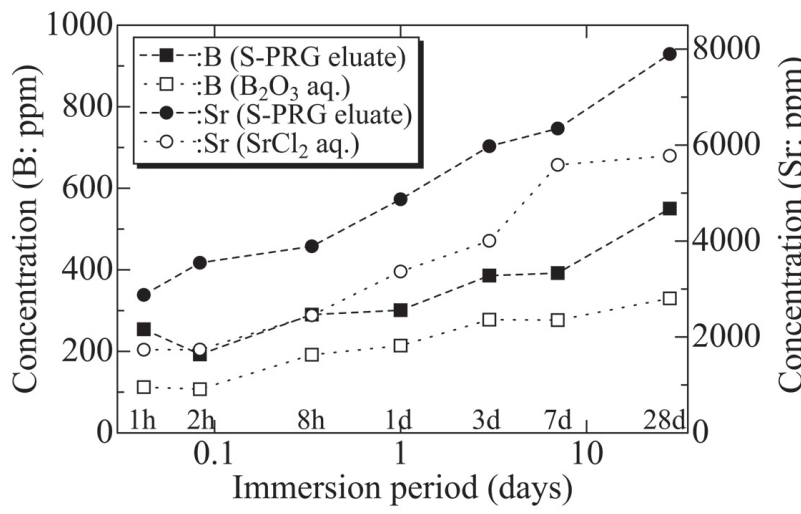

Fig. 2 Comparison of the time dependence (logarithmic) of the changes in Sr and B concentrations in enamel immersed in the S-PRG eluate, Sr solution, and B solution.

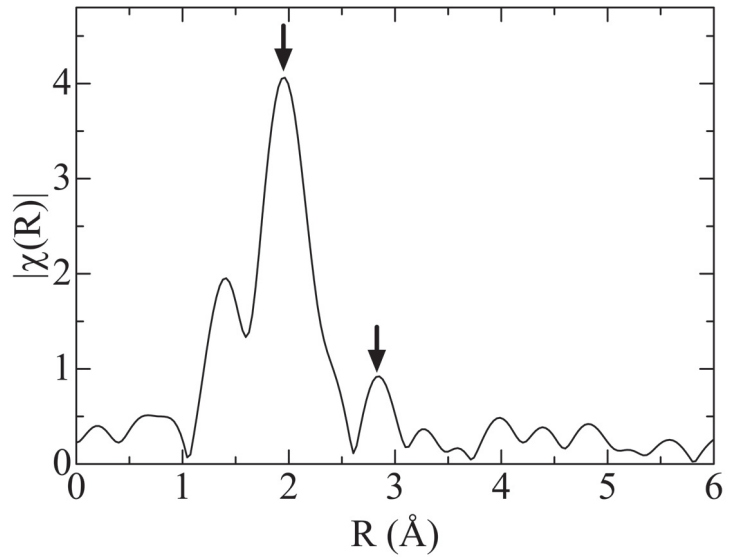

Fig. 3 The radial distribution function (RDF) of enamel immersed in S-PRG eluate for 1 day. 
Table 3 Comparison of the B and Sr concentration in the enamel immersed in the S-PRG eluate and Sr or B solution for various period (standard deviation is indicated in parentheses)

\begin{tabular}{|c|c|c|c|c|}
\hline \multirow{2}{*}{ Immersion period } & \multicolumn{2}{|c|}{ B concentration (ppm) } & \multicolumn{2}{|c|}{ Sr concentration (ppm) } \\
\hline & S-PRG eluate & B solution & S-PRG eluate & Sr solution \\
\hline $1 \mathrm{~h}$ & $254(27)$ & $112(28)$ & $2,880(138)$ & $1,730(170)$ \\
\hline $2 \mathrm{~h}$ & $193(14)$ & 107 (6) & $3,550(30)$ & $1,740(38)$ \\
\hline $8 \mathrm{~h}$ & $290(10)$ & $192(9)$ & $3,890(108)$ & $2,450(52)$ \\
\hline 1 days & $301(26)$ & $214(8)$ & $4,870(186)$ & $3,360(416)$ \\
\hline 3 days & 386 (16) & $278(16)$ & $5,980(188)$ & $4,000(39)$ \\
\hline 7 days & $392(11)$ & $277(7)$ & $6,350(76)$ & $5,590(227)$ \\
\hline 28 days & $550(14)$ & $330(9)$ & $7,900(255)$ & $5,780(77)$ \\
\hline
\end{tabular}

Table 4 Molar concentration of $\mathrm{Al}, \mathrm{B}$, and $\mathrm{Sr}$ in the S-PRG eluate and the enamel immersed in the S-PRG eluate for 28 days

\begin{tabular}{lccc}
\hline \multicolumn{1}{c}{ Specimens } & \multicolumn{3}{c}{ Molar concentration of elements } \\
\cline { 2 - 4 } & $\mathrm{Al}$ & $\mathrm{B}$ & $\mathrm{Sr}$ \\
\hline $\begin{array}{l}\text { S-PRG eluate } \\
(\mathrm{mmol} / \mathrm{L})\end{array}$ & 0.97 & 155 & 1.70 \\
$\begin{array}{l}\text { Immersed enamel (after 28 days) } \\
(\mu \mathrm{mol} / \mathrm{g})\end{array}$ & 50.0 & 50.9 & 90.2 \\
\hline
\end{tabular}

as well as their coordination. The peaks were observed at approximately 2 and $2.8 \AA$, which may have been derived from the $\mathrm{Sr}-\mathrm{O}$ first shell and $\mathrm{Sr}-\mathrm{P}$ second shell interactions (arrowed in Fig. 3).

\section{DISCUSSION}

This study evaluated the time dependence of the incorporation of multiple ions from S-PRG filler eluate into human enamel powder. For ions present in S-PRG eluate, Al, B (borate), and $\mathrm{Sr}$ ions were incorporated into enamel, as shown in Table 2 and Fig. 1A. In contrast, $\mathrm{Na}$ concentration in enamel did not change upon immersion, as shown in Fig. 1B. The initial concentrations of Al, B, and $\mathrm{Sr}$ in enamel were comparable to ( $\mathrm{Al}$ and $\mathrm{Sr}$ ) or lower than (B) those in the S-PRG eluate. Therefore, those ions would be easily incorporated (i.e., from the eluate into enamel). In contrast, the initial concentration of $\mathrm{Na}$ in enamel was quite high compared with that in the eluate; therefore, $\mathrm{Na}$ incorporation from the eluate would not readily occur. Similarly, concentrations of $\mathrm{Mg}$ and $\mathrm{Zn}$ in enamel, which are not present in S-PRG eluate, did not change upon immersion.

The slope of Fig. 1A indicates the rate of incorporation into enamel for each ion. $\mathrm{Al}$ and $\mathrm{Sr}$ showed the highest rates of incorporation into enamel, while $\mathrm{B}$ showed the lowest comparative rate. The concentrations of $\mathrm{Al}, \mathrm{B}$, and $\mathrm{Sr}$ in S-PRG eluate were 26, 1,680, and 149 ppm, respectively, as shown in Table 1; the final concentrations of those ions in enamel after 28 days of immersion were 1,350, 550, and 7,900 ppm, respectively, as shown in Table 2. The molar concentrations of Al, B, and $\mathrm{Sr}$ in S-PRG eluate and the concentrations of ions incorporated in enamel after 28 days of immersion were estimated as shown in Table 4. The concentrations of $\mathrm{Sr}$ and $\mathrm{Al}$ in S-PRG eluate were lower than that of B; notably, those were effectively absorbed from S-PRG eluate and incorporated into enamel. Although B concentration in S-PRG eluate was two-fold higher than that of $\mathrm{Sr}$ and $\mathrm{Al}$, the incorporation of $\mathrm{B}$ in enamel was lower than that of $\mathrm{Sr}$ and similar to that of $\mathrm{Al}$. Therefore, the incorporation of B occurred slowly, compared with that of $\mathrm{Al}$ and $\mathrm{Sr}$.

The different behaviors with respect to incorporation of $\mathrm{Al}, \mathrm{B}$, and $\mathrm{Sr}$ could be explained on the basis of their ionic radii, as follows. $\mathrm{Al}$ and $\mathrm{Sr}$ ions are monoatomic ions $\left(\mathrm{Al}^{3+}\right.$ and $\left.\mathrm{Sr}^{2+}\right)$, while $\mathrm{B}$ forms a molecular ion $\left(\mathrm{BO}_{3}{ }^{3-}\right)$. The ionic radii of $\mathrm{Al}^{3+}, \mathrm{Sr}^{2+}$, and $\mathrm{BO}_{3}{ }^{3-}$ are reported as 53, 118 , and $191 \mathrm{pm}$, respectively ${ }^{20,21)}$. The ionic radius of $\mathrm{BO}_{3}{ }^{3-}$, which is a molecular ion, is larger than the radii of the monoatomic $\mathrm{Al}^{3+}$ and $\mathrm{Sr}^{2+}$ ions. The internal diffusion of absorbed ions in the enamel matrix is related to ionic radius; faster diffusion is expected for monoatomic ions. Therefore, faster rates of incorporation would be reasonable for $\mathrm{Al}$ and $\mathrm{Sr}$.

Notably, Sr ion incorporation occurred efficiently, such that the final concentration in enamel reached 7,900 ppm after 28 days of immersion, although the 
concentration in the immersed S-PRG eluate was $149 \mathrm{ppm}$. In addition, nearly one-third of the final concentration of $\mathrm{Sr}$ (i.e., 2,880 ppm) was incorporated after $1 \mathrm{~h}$ of immersion. Fast Sr absorption was previously reported by Nakamura et al. ${ }^{14)}$; in that report, slight $\mathrm{Sr}$ adsorption occurred on the bulk enamel surface after less than $1 \mathrm{~h}$ of contact with an S-PRG containing experimental toothpaste. In the present study, Sr ions were supplied in S-PRG filler eluate; enamel powder was used as the test substance; therefore, Sr incorporation occurred more rapidly than in the report by Nakamura et al. In addition, rapid incorporation of other ions (e.g., $\mathrm{Al}$ and borate) was observed, similar to that of $\mathrm{Sr}$ after $1 \mathrm{~h}$ of immersion for the S-PRG eluate. Therefore, the incorporation of multiple ions could be expected at early stages of contact or exposure to S-PRG filler-containing dental materials, or to their eluate.

Regarding the local structure of the incorporated $\mathrm{Sr}$, it was estimated from the RDF around $\mathrm{Sr}$ in enamel immersed in S-PRG eluate for 1 day (a relatively early stage of S-PRG eluate immersion), as shown in Fig. 3. One of the authors had reported the RDF of Sr in S-PRG eluate-immersed enamel for 3 months and $\mathrm{Sr}$ was suspected to be incorporated into the Ca site of the HAP crystal in enamel ${ }^{19}$. Due to the similarity of RDFs for specimens after 1 day and 3 months of immersion, Sr after 1 day of immersion in S-PRG eluate was expected to be in the same state after 3 months of immersion. Then, $\mathrm{Sr}$ absorbed from S-PRG eluate is likely to be incorporated into the Ca site of HAP in the early stage of immersion. The $\mathrm{Sr}$ ion is divalent, similar to $\mathrm{Ca}$; the ionic radius of $\mathrm{Sr}^{2+}(118 \mathrm{pm})$ is slightly larger than, but compatible with, that of $\mathrm{Ca}^{2+}(100 \mathrm{pm})$. Therefore, $\mathrm{Sr}$ incorporation is likely to readily occur, such that the final concentration of Sr would be higher than the concentrations of other ions in this study. The ionic radius of $\mathrm{Al}^{3+}(53 \mathrm{pm})$ is smaller than that of $\mathrm{Ca}^{2+}$; moreover, its ionic valence differs. Therefore, Al incorporation is relatively less likely to occur.

In comparison of the ion incorporation behavior, as shown in Fig. 2, both cation $\left(\mathrm{Sr}^{2+}\right)$ and anion (borate) showed greater incorporation into enamel with S-PRG eluate immersion, compared with immersion in the $\mathrm{Sr}$ and B solutions. This simultaneous ion incorporation from S-PRG eluate likely occurred because the cation/ anion charge was well-balanced; thus, ion incorporation readily occurred. In the Sr solution, the counterion $\left(\mathrm{Cl}^{-}\right)$was seldom incorporated because of its large ionic radius (184 pm). In the $\mathrm{B}$ solution, no cation was present except for $\mathrm{H}^{+}$; here, an imbalance in the charge due to incorporated ions would inhibit further ion incorporation. Therefore, simultaneous multiple ion incorporation with S-PRG eluate is suggested.

Importantly, S-PRG eluate contains high concentrations of multiple cations and anions, which are present in stable forms. Some of these ions $\left(\mathrm{Sr}^{2+}\right.$, $\left.\mathrm{BO}_{3}{ }^{3-}, \mathrm{F}^{-}\right)$are expected to demonstrate bioactive effects. Simultaneous incorporation of ions into enamel might be enhanced by balancing their charges, compared with applications of single ion solutions.

\section{CONCLUSION}

In this study, the time-dependent incorporation of ions from S-PRG eluate into tooth enamel was estimated by using ICP-AES. Al, B, and Sr concentrations in enamel increased in a time-dependent manner upon immersion in S-PRG eluate. Those ions were quickly incorporated, such that they reached half of their final concentrations (final concentrations measured at $28 \mathrm{~h}$ ) after $2 \mathrm{~h}$ of immersion. Despite relatively low concentrations of $\mathrm{Al}$ and $\mathrm{Sr}$ in S-PRG eluate, those ions were effectively incorporated into the enamel. In contrast, B showed lower incorporation although it exhibited the highest concentration in S-PRG eluate. This difference in incorporation behavior could be a function of the specific ionic species and radii involved. Compared with single-ion solutions ( $\mathrm{Sr}$ and $\mathrm{B}$ ), ions were incorporated at high concentrations upon immersion in S-PRG eluate. The simultaneous incorporation of cations and anions under balanced charge conditions may facilitate effective incorporation. Thus, various useful ions may be effectively incorporated into tooth enamel by applying S-PRG filler or its eluate; greater bioactive effects might occur.

\section{ACKNOWLEDGMENTS}

This work was supported by a Grant-in Aid from the Japan Society for the Promotion of Science (JSPS No. 16H02688). EXAFS measurements were performed with the approval of the Photon Factory Program Advisory Committee (Proposal No.2015G557).

\section{REFERENCES}

1) Aoba T. Solubility properties of human tooth mineral and pathogenesis of dental caries. Oral Dis 2004; 10: 249-257.

2) ten Cate JM. Fluorides in caries prevention and control: Empiricism or science. Caries Res 2004; 38: 254-257.

3) Curzon MEJ, Losee FL. Strontium content of enamel and dental caries. Caries Res 1977; 11: 321-326.

4) Happonen SS, Luoma H, Forss H, Kentala J, Alaluusua $\mathrm{S}$, Luoma $\mathrm{AR}$, et al. Effects of a chlorhexidine-fluoridestrontium rinsing program on caries, gingivitis and some salivary bacteria among Finnish school children. Eur J Oral Res 1991; 99: 130-138.

5) Fujimoto Y, Iwasa M, Murayama R, Miyazaki M, Nagafuji A, Nakatsuka T. Detection of ions released from S-PRG fillers and their modulation effect. Dent Mater J 2010; 29: 392-397.

6) Hotta M, Morikawa T, Tamura D, Kusakabe S. Adherence of Streptococcus sanguinis and Streptococcus mutans to salivacoated S-PRG resin blocks. Dent Mater J 2014; 33: 261-267.

7) Nomura R, Morita Y, Matayoshi S, Nakano K. Inhibitory effect of surface pre-reacted glass-ionomer (S-PRG) eluate against adhesion and colonization by Streptococcus mutans. Sci Rep 2018; 8: 5056.

8) Miki S, Kitagawa H, Kitagawa R, Kiba W, Hayashi M, Imazato S. Antibacterial activity of resin composites containing surface pre-reacted glass-ionomer (S-PRG) filler. Dent Mater 2016; 32: 1095-1102.

9) Kitagawa H, Miki-Oka S, Mayanagi G, Abiko Y, Takahashi $\mathrm{N}$, Imazato S. Inhibitory effect of resin composite containing S-PRG filler on Streptococcus mutans glucose metabolism. J Dent 2018; 70: 92-96. 
10) Yoneda M, Suzuki N, Masuo Y, Fujimoto A, Iha K, Yamada K, et al. Effect of S-PRG eluate on biofilm formation and enzyme activity of oral bacteria. Int J Dent 2012; 2012: 814913.

11) Suzuki N, Yoneda M, Haruna K, Masuo Y, Nishihara T, Nakanishi K, et al. Effects of S-PRG eluate on oral biofilm and oral malodor. Arch Oral Biol 2014; 59: 407-413.

12) Mukai Y, Kamijo K, Fujino F, Hirata Y, Teranaka T, Ten Cate JM. Effect of denture base-resin with prereacted glass ionomer filler on dentin demineralization. Eur J Oral Sci 2009; 117: 750-754.

13) Ma S, Imazato S, Chen J, Mayanagi G, Takahashi N, Ishimoto $\mathrm{T}$, et al. Effects of a coating resin containing S-PRG filler to prevent demineralization of root surfaces. Dent Mater J 2012; 31: 909-915.

14) Nakamura K, Hamba H, Nakashima S, Sadr A, Nikaido T, Oikawa M, et al. Effects of experimental pastes containing surface pre-reacted glass ionomer fillers on inhibition of enamel demineralization. Dent Mater J 2017; 36: 482-490.

15) Ito S, Iijima M, Hashimoto M, Tsukamoto N, Mizoguchi I, Saito T. Effects of surface prereacted glass-ionomer fillers on mineral induction by phosphoprotein. J Dent 2011; 39: 72 -
79 .

16) Iijima M, Ito S, Nakagaki S, Kohda N, Muguruma T, Saito $\mathrm{T}$, et al. Effects of immersion in solution of an experimental toothpaste containing SPRG filler on like-remineralizing ability of etched enamel. Dent Mater J 2014; 33: 430-436.

17) Han L, Okiji T. Evaluation of the ions release/incorporation of the prototype S-PRG filler containing endodontic sealer. Dent Mater J 2011; 30: 898-903.

18) Funato $Y$, Matsuda $Y$, Okuyama K, Yamamoto H, Komatsu $\mathrm{H}$, Sano H. A new technique for analyzing trace element uptake by human enamel. Dent Mater J 2015; 34: 240-245.

19) Uo M, Wada T, Asakura K. Structural analysis of strontium in human teeth treated with surface pre-reacted glass-ionomer filler eluate by using extended X-ray absorption fine structure analysis. Dental Mater J 2017; 36: 214-221.

20) Wired Chemist [Internet]: Metallic, Covalent and Ionic Radii(r)* [cited 2018 Sept 1]. Available from: http://www. wiredchemist.com/chemistry/data/metallic-radii

21) Wired Chemist [Internet]: Thermochemical Radii-Anions [cited 2018 Sept 1]. Available from: http://www.wiredchemist. com/chemistry/data/thermochemical-radii-anions 